\title{
Relationship between Length and Surface-Enhanced Raman Spectroscopy Signal Strength in Metal Nanoparticle Chains: Ideal Models versus Nanofabrication
}

\author{
Kristen D. Alexander, ${ }^{1}$ Shunping Zhang, ${ }^{2}$ Angela R. Hight Walker, ${ }^{3}$ \\ Hongxing $\mathrm{Xu}^{2}{ }^{2}$ and Rene Lopez ${ }^{1}$ \\ ${ }^{1}$ Department of Physics and Astronomy, University of North Carolina, Chapel Hill, NC 27599, USA \\ ${ }^{2}$ Beijing National Laboratory for Condensed Matter Physics, Institute of Physics, Chinese Academy of Sciences, Beijing 100190, China \\ ${ }^{3}$ Optical Technology Division, National Institute of Standards and Technology, Gaithersburg, MD 20899, USA
}

Correspondence should be addressed to Rene Lopez, rln@physics.unc.edu

Received 5 November 2011; Accepted 18 January 2012

Academic Editor: Mustafa Çulha

Copyright (C) 2012 Kristen D. Alexander et al. This is an open access article distributed under the Creative Commons Attribution License, which permits unrestricted use, distribution, and reproduction in any medium, provided the original work is properly cited.

We have employed capillary force deposition on ion beam patterned substrates to fabricate chains of 60 nm gold nanospheres ranging in length from 1 to 9 nanoparticles. Measurements of the surface-averaged SERS enhancement factor strength for these chains were then compared to the numerical predictions. The SERS enhancement conformed to theoretical predictions in the case of only a few chains, with the vast majority of chains tested not matching such behavior. Although all of the nanoparticle chains appear identical under electron microscope observation, the extreme sensitivity of the SERS enhancement to nanoscale morphology renders current nanofabrication methods insufficient for consistent production of coupled nanoparticle chains. Notwithstanding this fact, the aggregate data also confirmed that nanoparticle dimers offer a large improvement over the monomer enhancement while conclusively showing that, within the limitations imposed by current state-of-the-art nanofabrication techniques, chains comprising more than two nanoparticles provide only a marginal signal boost over the already considerable dimer enhancement.

\section{Introduction}

Scientific interest in surface-enhanced Raman spectroscopy (SERS) has increased dramatically since the detection of single molecules was first reported in 1997 [1]. While enhancement factors measured from bulk SERS-active materials typically hover around $10^{6}[2,3]$, researchers have succeeded in designing "hot spots" on substrates capable of producing values over $10^{8}$ (on the order of the level necessary for detecting single molecules) [4-7]. The prospect of being able to boost sensitivity of SERS measurements at the single molecule level coupled with the fingerprint-like uniqueness of the Raman spectrum makes SERS a sought-after candidate for incorporation into sensor technologies; particularly, for those who are interested in detecting chemical compounds at the trace level.
Although researchers have discovered many nanoscaletextured metallic surfaces capable of producing the strong electromagnetic fields that give rise to SERS enhancements, metal nanoparticle aggregates have long been recognized as ideal materials for the achievement of truly large enhancement factors. Among these, the simplicity of the spherical nanoparticle dimer has made it a structure of choice in many attempts to build arrays of highly sensitive and reproducible SERS-active structures [8]. In a recent study, however, the question has been posed as to whether the two-particle linear aggregate is, indeed, the optimal configuration for generating SERS enhancements, prompting researchers to consider other morphologies [9]. One such configuration is closely spaced metal nanoparticles arranged end to end to form a chain (a natural extension of the dimer configuration). This structure was theoretically investigated by Wang et al. [9], 
who carried out finite integral technique (FIT) simulations on gold and silver nanoparticle chains comprised of even numbers of nanoparticles ranging in length from 2 nanoparticles to infinity. The results of the simulations indicate that the field enhancement at the gap between the two centermost particles (Wang refers to this as the "c-point") can reach a maximum at a number larger than two, suggesting that the dimer might not always be the ideal configuration for achieving the maximum field enhancement. In contrast to these predictions, recent theoretical and experimental results by Wustholz et al. [10] suggest that, in most realistic experiments, the signal from the hottest hot spot in a cluster is often extremely dominant and, thus, renders unrealistic the possibility of enhancement due to near-field coupling along the chain as a whole. In this paper, the main aim is to test these conclusions on precisely designed nanoparticle chains. Through the combination of focused ion beam (FIB) milling and capillary forced deposition [11], closely spaced linear nanoclusters were fabricated. The SERS enhancement arising from these structures were measured and subsequently compared with theoretically predicted values.

\section{Generalized Mie Theory Simulations}

Because it would not be possible for us to measure the SERS enhancement factor (EF) directly at the c-point, we have carried out generalized Mie theory (GMT) [12-15] simulations to map the electromagnetic strength over the entire surface of gold nanospheres chains ranging in length from 1 to 9 nanoparticles with interparticle separations of $1-1.5 \mathrm{~nm}$ (these separations still yield reliable results in the frame of classical electromagnetic theory).

The surface Raman enhancement is proportional to the product of the squares of the field enhancement at the incident and Raman frequencies,

$$
G=\left|f\left(\omega_{\text {Raman }}\right)\right|^{2}\left|f\left(\omega_{\text {pump }}\right)\right|^{2} .
$$

Provided that $\left|\omega_{\text {Raman }}-\omega\right|$ is smaller than the spectral response of the metal nanostructure and the excitation source is polarized along the axis of the chain, the SERS enhancement can be approximated to scale with the fourth power of the electric field enhancement [16]:

$$
G(r) \approx \frac{\left|E_{\mathrm{loc}}(r)\right|^{4}}{\left|E_{0}(r)\right|^{4}}
$$

Since the molecular species to be detected is assumed to form a monolayer on the surface of the nanospheres, the Raman EF must be averaged over the entire chain surface

$$
\langle G(r)\rangle=\frac{1}{\sum_{i}^{N} 4 \pi R_{i}^{2}} \sum_{i}^{N} \int_{i} G(r) d \sigma,
$$

where $N$ is the number of nanoparticles in the chain, $R_{i}$ is the radius of the $i$ th nanoparticle, and the fields were calculated at a distance of $0.5 \mathrm{~nm}$ from the surface which, based on the estimated thickness of the benzenethiol monolayer (the reporter molecule used in the experimental part of this study), is the most likely location of the reporter molecule. Here, it is important to note that, although the length of the benzenethiol molecule and bond length on gold are known, the interparticle distance for the structures fabricated in this experiment cannot be known exactly. Similarly, a monolayer of thiophenol molecules surrounding the spheres and the silica/silicon substrate invariably alters the index of refraction of the area immediately surrounding the metal surface. To account for this range in gap size and the effective index of refraction of the surrounding medium, $n_{\text {eff }}$, we calculated the surface averaged enhancement for a gap of $1 \mathrm{~nm}$ and $n_{\text {eff }}=1.0$ (Figure $1(\mathrm{a})$ ) as well as for a gap of $1.5 \mathrm{~nm}$ and $n_{\text {eff }}=1.5$ (Figure $1(\mathrm{~b})$ ). As expected, the surface Raman enhancements of the nanochains $(N \geq 2)$ reach values on the order of $10^{7}-10^{8}$ over that of the monomer at resonance. Furthermore, the plasmon resonances shift to longer wavelengths as the particle number increases. Figures 1(c) and 1(d) show the SERS enhancement at the laser line $(632.8 \mathrm{~nm})$ as a function of particle number, corresponding to Figures 1(a) and 1(b), respectively. Significantly, a peak is apparent in the SERS enhancement at a chain length of 3 nanoparticles for the $1 \mathrm{~nm}$ gap $\left(n_{\mathrm{eff}}=1.0\right)$ and 2 nanoparticles for the $1.5 \mathrm{~nm}$ gap $\left(n_{\mathrm{eff}}=1.5\right)$. It is important to clarify that this simulation approach was designed to produce results that could be compared to experimental measurements of the SERS EF where reporter molecules actually coat all metallic surfaces. This is in contrast to the FIT calculations performed by Wang et al. which by focusing in the field enhancement at the "c-point" cannot be directly compared to a far-field experimental measurement.

\section{Finite Difference Time Domain (FDTD) Simulations}

To identify the link between nanoparticle chain length and the location of the surface Raman enhancement peak, we also calculated the extinction spectrum for the linear chains. As our GMT code is designed for near-field calculations, we have rather turned to apply a fast and commercially available finite difference time domain (FDTD) method (Lumerical Solutions, Inc) for far-field results. While FDTD cannot compete with the accuracy of GMT for the near field calculations, it is a perfect alternative in the far field. In order to ensure accuracy, simulations were carried out with a mesh override region surrounding the entire structure with cell size limited to $0.8 \mathrm{~nm}$ and convergence of the results were confirmed by comparing the results of multiple runs.

Results from these simulations are shown in Figure 2. First, the redshift of the extinction peak with respect to the increased particle number is apparent, indicating as expected, the tight correlation between the optical response and the morphology of the scatterer. Most importantly, however, the location of the highest peak of the extinction with respect to wavelength coincides with the highest EF in the near-field calculations in Figure 1. Here, we note that when we narrow our observations to a fixed wavelength 


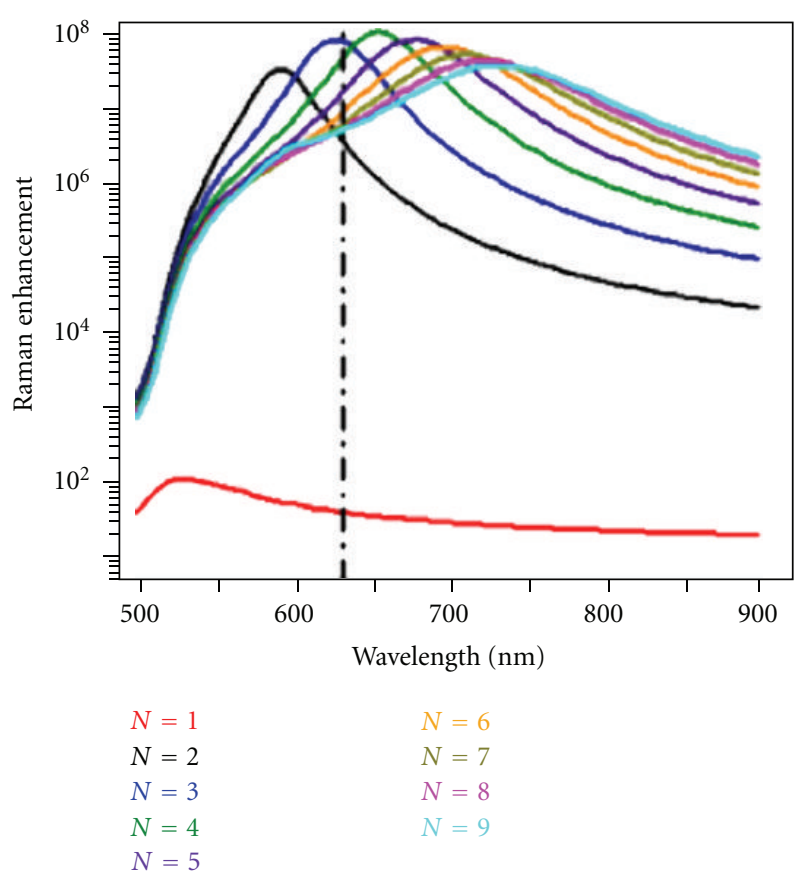

(a)

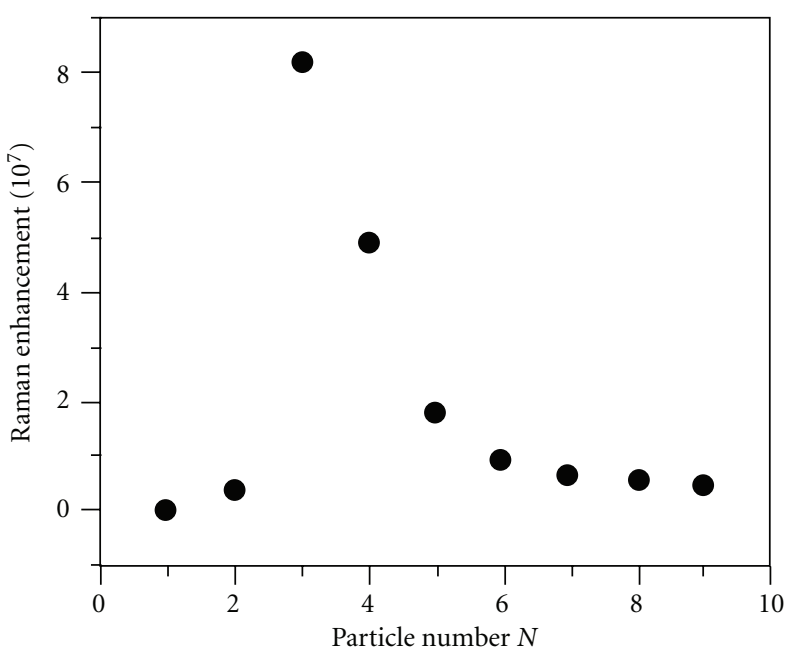

(c)

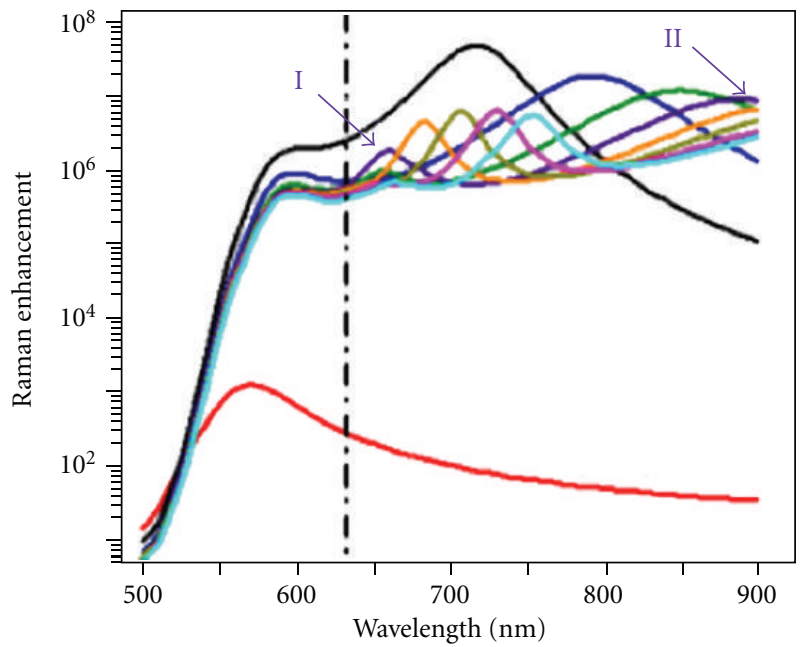

$\begin{array}{ll}N=1 & N=6 \\ N=2 & N=7 \\ N=3 & N=8 \\ N=4 & N=9 \\ N=5 & \end{array}$

(b)

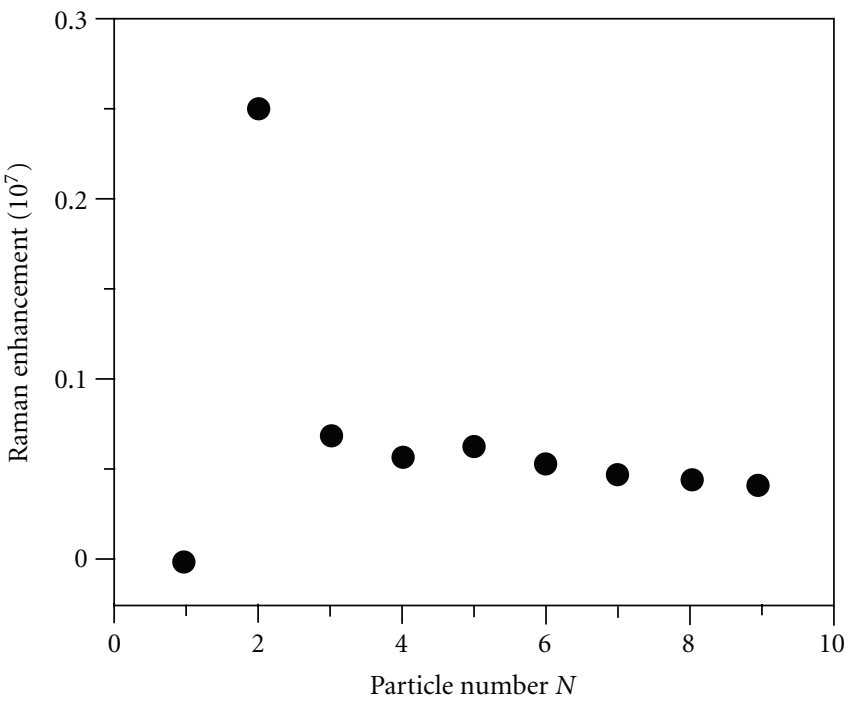

(d)

FIgURE 1: Calculated SERS EFs for Au nanoparticle chains using generalized Mie theory. The EF spectra have been calculated for chains ranging 1-9 nanoparticles in length with excitation source parallel to the long axis of the chain for an interparticle gap of $1 \mathrm{~nm}$ and $n_{\text {eff }}=1.0$ (a) and an interparticle gap of $1.5 \mathrm{~nm}$ and $n_{\text {eff }}=1.5$ (b). The EF for the $632.8 \mathrm{~nm}$ excitation source for (a) and (b) are shown, respectively, in (c) and (d). Angular momentum contributions up to the 37 th order were included in the GMT calculations. The integral was taken at a distance of $0.5 \mathrm{~nm}$ from the particle surface for (a) and $0.75 \mathrm{~nm}$ from the particle surface for (b). The two enhancement peaks for the $N=5$ chain are identified as II and I, respectively, in (b).

(633 $\mathrm{nm}$ in this case) small secondary peaks become relevant, making the direct comparison between the far and near fields less direct. Figures 1(c) and 1(d) plot the EF magnitude at the fixed wavelength againts the number of particles in each chain. One can observe that the EF peaks at a particular number of particles, and that number is not necessarily two (Figure 1(c)).

\section{Sample Nanofabrication}

To experimentally test the simulation results, a templating method was employed to create nanoparticle chains of specific lengths and orientations. FIB milling was used to create trenches in $\mathrm{SiO}_{2}$ which were approximately $80 \mathrm{~nm}$ in width, $70 \mathrm{~nm}$ in depth, and ranging from 80 to $560 \mathrm{~nm}$ 


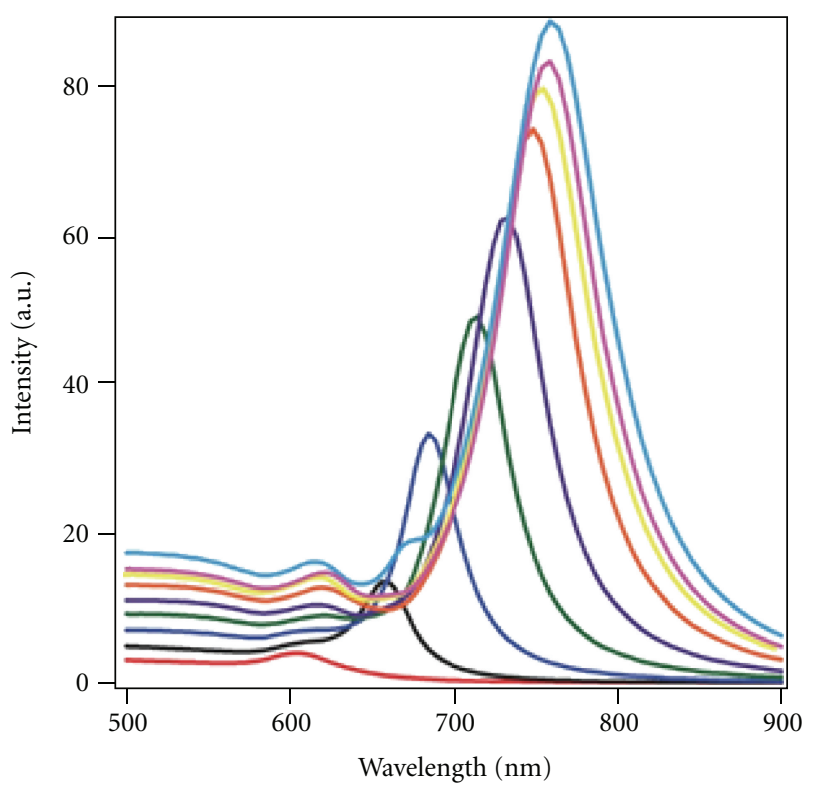

(a)

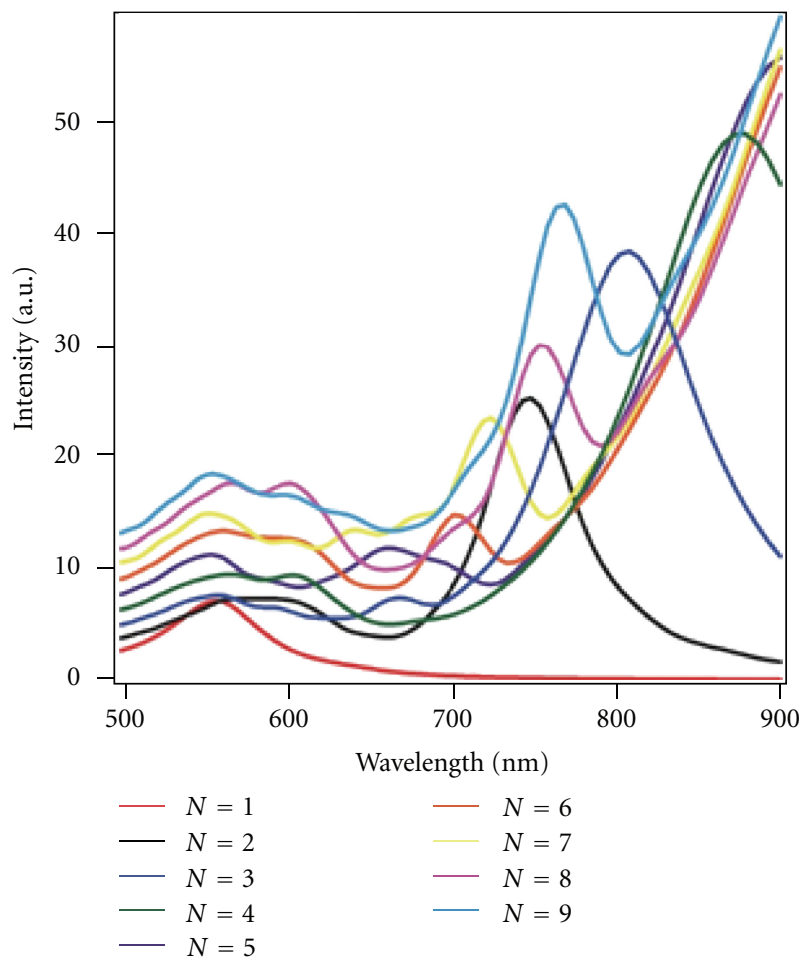

(b)

FIgURE 2: FDTD calculations of the extinction spectra for $60 \mathrm{~nm}$ $\mathrm{Au}$ nanoparticle chains 1-9 nanoparticles in length. Nanochains in (a) have a $1 \mathrm{~nm}$ interparticle separation and are embedded in a medium with refractive index $n_{\text {eff }}=1.0$. Nanochains in (b) have a $1.5 \mathrm{~nm}$ interparticle separation and are embedded in a medium with refractive index $n_{\text {eff }}=1.5$.

in length. Gold nanospheres (Ted Pella) with a diameter of $60 \mathrm{~nm}$ were placed into these trenches using a capillary force deposition method [11]. To accomplish this, the nanopatterned substrate was cleaned in piranha solution and subsequently subjected to a brief plasma treatment to ensure the hydrophilicity of the $\mathrm{SiO}_{2}$ layer. Next, the $\mathrm{SiO}_{2}$ was immersed in an aqueous gold colloid which was allowed to evaporate at $70^{\circ}$ overnight. As the meniscus slowly recedes across the substrate, nanoparticles concentrate at the three-phase contact line (Figure 3). Because the trenches are designed to host only a specific number and configuration of nanoparticles, this process creates arrays of nanoparticle chains of predetermined lengths. Scanning-electron micrographs of the substrates were taken to identify satisfactory chains. To remove carbonaceous contamination that often accompanies SEM imaging, substrates were subsequently cleaned with piranha solution and treated with $\mathrm{O}_{2}$ plasma for 10 minutes to remove any unwanted material that the electron beam might have deposited. Finally, nanochains were immersed in a $10 \mathrm{mM}$ solution of benzenethiol overnight, removed, rinsed for 60 seconds with ethanol to remove excess benzenethiol, and then blown dry with $\mathrm{N}_{2}$.

\section{Raman Measurements}

Raman spectra of individual chains were obtained using a Leica microscope equipped with a confocal Raman spectroscopic system (Renishaw InVia) and a $30 \mathrm{~mW} 632.8 \mathrm{~nm}$ laser excitation source. All spectra were measured using a $50 \times$ objective with a numerical aperture of 0.75 . The Raman signal was collected by a TE air-cooled $576 \times$ 400 CCD array preceded by two notch filters (OD > 12) to block the laser line. Preliminary tests concluded that, without sufficient filtering, persistent exposure to the laser beam causes the benzenethiol monolayer to degrade. To avoid photodegradation of the monolayer, the stability of the Raman signal was monitored over a range of beam powers and exposure times. These tests determined that the combination of a $100 \mu \mathrm{W}$ laser beam power (spot size diameter $\sim 2 \mu \mathrm{m}$ ) delivered on the sample with a 30 -second acquisition time yielded sufficiently clear and stable spectra. To account for the possibility that the laser spot was not perfectly aligned over the chain and/or not perfectly uniform, a flat scanning stage was used to raster over the structure. From these measurements, the strongest, most well-defined spectrum from each of the structures was selected for data analysis. A typical spectrum for a nanoparticle heptamer is shown in Figure 4. The most pronounced peaks appear at $1000 \mathrm{~cm}^{-1}$ (ring out-of-plane deformation and C-H out-ofplane bending), $1080 \mathrm{~cm}^{-1}$ (C-C symmetric stretching and $\mathrm{C}-\mathrm{S}$ stretching), and $1577 \mathrm{~cm}^{-1}$ (C-C symmetric stretching) [17]. Time-stability tests were performed in order to select the most stable Raman peak for comparison in these tests. All the peaks were clear and steady, but the $1080 \mathrm{~cm}^{-1}$ peak was found to show the best signal to noise ratio and was, thus, used for all data analyses performed in this study.

\section{Summary of Results and Discussion}

Experimental results are plotted over GMT simulations for a $1.5 \mathrm{~nm}$ interparticle gap in Figure 5. Results from two data sets are shown to delineate the range of outcomes 


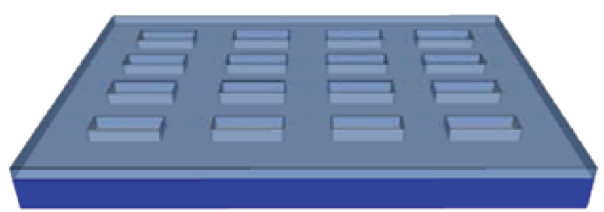

(a)

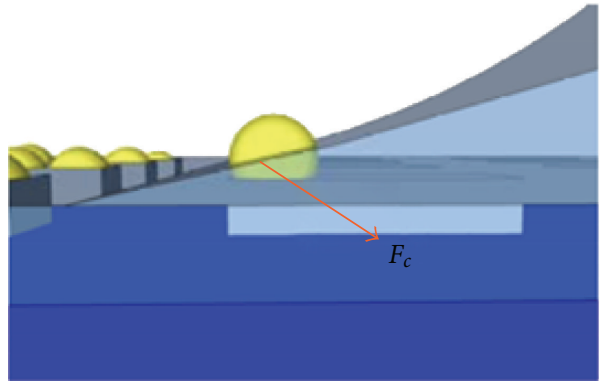

(c)

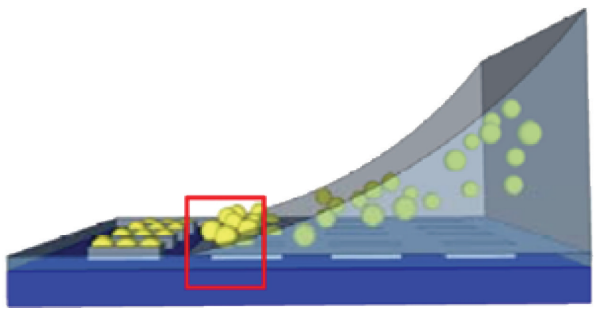

(b)

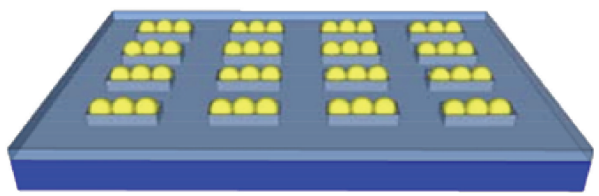

(d)

FIGURE 3: (a) Silicon substrate immediately after FIB milling of $\mathrm{SiO}_{2}$ layer. (b) Illustration of three-phase contact line dragging nanoparticles across the substrate surface. (c) Close-up illustration of particles pushed into milled trenches via the capillary force. (d) Array of nanosphere trimers after deposition is complete.

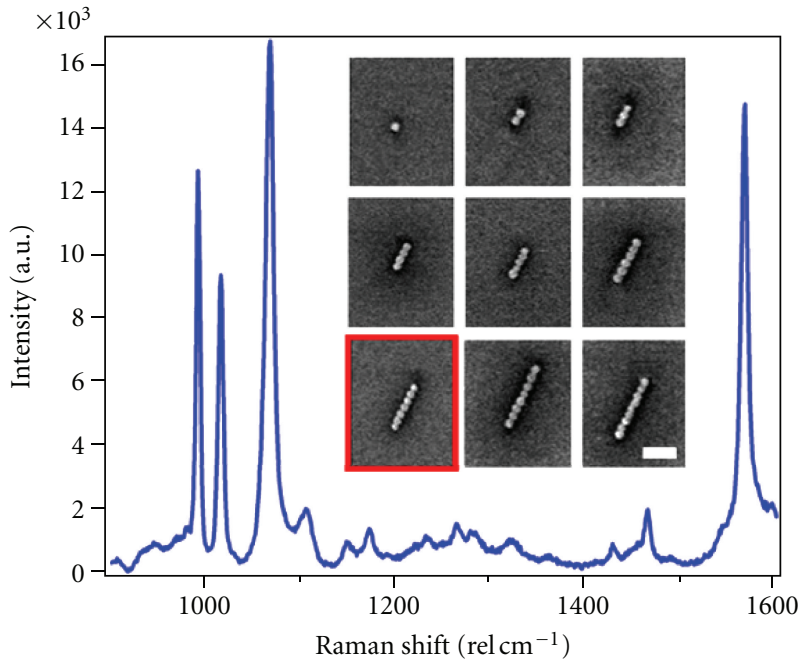

FIGURE 4: (a) Scanning-electron micrograph of gold nanochains ranging 1-9 particles in length. Scale bar is $300 \mathrm{~nm}$. (b) SERS spectrum of benzenethiol on a nanochain comprised of $60 \mathrm{~nm}$ gold nanospheres. Measurements were taken with a $632.8 \mathrm{~nm}$ laser source polarized parallel to the nanochain axis. The red box in (a) denotes the heptamer from which this measurement was taken.

for this particular experiment. Specifically, the first set represents the largest measured enhancements (i.e., the best enhancements obtained in this study) for each chain length (red trace), and a second set with the results falling within one standard deviation of the overall data average (blue trace). It can be observed that the second set deviates from theory especially at longer chain lengths, reaching a plateau of enhancement rather than decreasing for larger particle numbers. The large enhancement ratio between the monomer and the dimer is unsurprising, as it is well known that the tiny gaps formed by closely spaced nanoparticles are the source of large electric fields which give rise to large SERS enhancement factors. This situation becomes more complex, however, when the number of gaps in the chain is increased through the addition of nanoparticles. It has been shown that the SERS EF is extremely sensitive to interparticle separation, falling off at the rate of approximately one order of magnitude per nanometer of separation [18]. For longer nanochains, a mixture of coupled and decoupled neighboring particles might coexist. Moreover, the extreme sensitivity of the SERS enhancement to a gamut of factors, including nanoparticle size [19], shape [20], crystal face [21], surface roughness [22], and particle-particle spacing [23-27] is well documented and is known to account for significant variations in EFs arising from seeminglyidentical nanoparticle clusters. Taking these two items into consideration, one can see that in practice it is unlikely that the enhancement that would arise from a perfectly coupled nanochain of a specific length is being observed. Moreover, it is quite likely that a single dominant hot spot is accounting for the majority of the enhancement observed for all chain lengths $>1$. Significantly, these considerations qualitatively explain the plateau of the EF in Figure 5 as well as emphasize the importance of making hot spots through gap formation over additional enhancements achieved through resonances in complex geometries, thereby supporting the Wustholz et al. [10] conclusion.

At the other end of the spectrum of experimental outcomes, the largest enhancement results do show a significant 


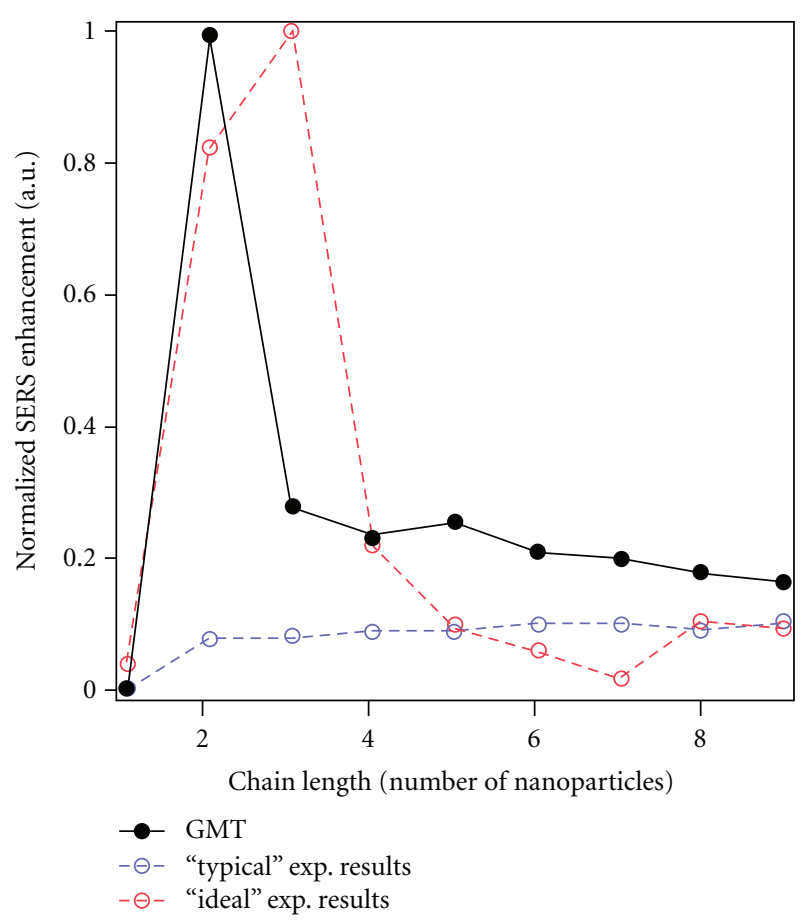

FIgURE 5: Black trace-generalized Mie theory calculations for the SERS EF for $60 \mathrm{~nm}$ diameter Au nanochains with a $1.5 \mathrm{~nm}$ interparticle separation, embedded in a medium with refractive index $n_{\text {eff }}=1.5$. Blue trace-experimental SERS EF measurements for $60 \mathrm{~nm}$ diameter $\mathrm{Au}$ nanochains lying within one standard deviation from the average measured EF value for each chain length (i.e., "typical" results). Red trace-largest values for the SERS EF for each chain length measured in this experiment (i.e., "ideal" results). "Typical" results (blue trace) are normalized against the values for the "ideal" results (red trace) to illustrate the difference in magnitude between the two experimental data sets.

agreement with theory. While it would be impossible to accurately determine whether the peak at a chain length of 3 nanoparticles arises from perfect interparticle coupling along the entire length of the chain, the measurement seems to indicate that this was the case and it is certainly relevant to include a discussion of this ideal situation. Referring back to Figure 1, despite a small redshift, the maximum SERS enhancement for the monomers depicted in Figures 1(a) and 1(b) is located around $525 \mathrm{~nm}$ and $570 \mathrm{~nm}$, respectively, corresponding to the far-field LSPR peak. For aggregates consisting of more than two spheres, the hybridized plasmon modes appear as a result of the interaction of primary plasmons in nearby spheres $[28,29]$. This coupling dramatically enhances the local electric field, creating an SERS enhancement, that is, orders of magnitude larger than that of a single sphere. In addition to lowering the bonding mode, hybridized plasmon modes of higher angular momentum are also excited by the wave polarized parallel to the long axis of the chain. As the chain length is increased, both EF peaks experience a redshift. These chains demonstrate a sufficiently strong dipole moment and are, thus, able to couple to far field radiation efficiently, thereby creating the peaks in the EF observed in both the simulation and experimental data.

\section{Conclusions}

In summary, GMT and FDTD simulations were performed to model the surface-averaged electromagnetic field enhancements arising from plasmonic coupling in closely spaced noble metal nanoparticle chains. The simulations suggest that there exists an "ideal" chain length capable of producing modest improvements on the typical dimer enhancement factor. To experimentally test the model, chains of $60 \mathrm{~nm}$ gold nanospheres were fabricated using a capillary force deposition technique. Subsequent Raman measurements made from these structures showed modest agreement with the GMT simulations in some cases and a complete lack of agreement in others. In general, the vast majority of the tested nanoparticle chains showed no more enhancement beyond the typical for a two-particle dimmer. From these results, it was concluded that it is unlikely that the individual nanoparticles comprising the chains made using the capillary force deposition method technique are consistently able to couple completely along the entire structure, thereby departing from the ideal situation modeled in the calculations performed in this study. However, comparison of experimental and theoretical results confirms that the formation of dimers provides a large improvement in signal strength over the monomer enhancement. While perfect plasmonic coupling of the ideal number of nanoparticles can provide improvement over the dimer enhancement, it is marginal under the best of circumstances and often introduces more difficulties in the fabrication process than is justifiable by the small boost in signal.

\section{Acknowledgments}

This work was supported in part by the Hartley Corporation Fellowship for Graduate Women in Science and the SPIE Graduate Student fellowship. The authors thank Dr. Richard Superfine for many useful conversations.

\section{References}

[1] K. Kneipp, Y. Wang, H. Kneipp et al., "Single molecule detection using surface-enhanced raman scattering (SERS)," Physical Review Letters, vol. 78, no. 9, pp. 1667-1670, 1997.

[2] J. Lu, D. Chamberlin, D. A. Rider, M. Liu, I. Manners, and T. P. Russell, "Using a ferrocenylsilane-based block copolymer as a template to produce nanotextured Ag surfaces: uniformly enhanced surface enhanced Raman scattering active substrates," Nanotechnology, vol. 17, no. 23, pp. 5792-5797, 2006.

[3] A. Gopinath, S. V. Boriskina, W. R. Premasiri, L. Ziegler, B. M. Reinhard, and L. D. Negro, "Plasmonic nanogalaxies: multiscale aperiodic arrays for surface-enhanced Raman sensing," Nano Letters, vol. 9, no. 11, pp. 3922-3929, 2009.

[4] J. A. Dieringer, K. L. Wustholz, D. J. Masiello et al., "Surface-enhanced Raman excitation spectroscopy of a single rhodamine 6G molecule," Journal of the American Chemical Society, vol. 131, no. 2, pp. 849-854, 2009. 
[5] E. C. le Ru, E. Blackie, M. Meyer, and P. G. Etchegoint, "Surface enhanced Raman scattering enhancement factors: a comprehensive study," Journal of Physical Chemistry C, vol. 111, no. 37, pp. 13794-13803, 2007.

[6] H. Xu, E. J. Bjerneld, M. Käll, and L. Börjesson, "Spectroscopy of single hemoglobin molecules by surface enhanced Raman scattering," Physical Review Letters, vol. 83, no. 21, pp. 43574360, 1999.

[7] E. C. le Ru, M. Meyer, E. Blackie, and P. G. Etchegoin, "Advanced aspects of electromagnetic SERS enhancement factors at a hot spot," Journal of Raman Spectroscopy, vol. 39, no. 9, pp. 1127-1134, 2008.

[8] J. P. Camden, J. A. Dieringer, Y. Wang et al., "Probing the structure of single-molecule surface-enhanced raman scattering hot spots," Journal of the American Chemical Society, vol. 130, no. 38, pp. 12616-12617, 2008.

[9] Z. B. Wang, B. S. Luk'yanchuk, W. Guo et al., "The influences of particle number on hot spots in strongly coupled metal nanoparticles chain," The Journal of Chemical Physics, vol. 128, no. 9, Article ID 094705, 2008.

[10] K. L. Wustholz, A. I. Henry, J. M. McMahon et al., "Structureactivity relationships in gold nanoparticle dimers and trimers for surface-enhanced raman spectroscopy," Journal of the American Chemical Society, vol. 132, no. 31, pp. 10903-10910, 2010.

[11] Y. Cui, M. T. Björk, J. A. Liddle, C. Sönnichsen, B. Boussert, and A. P. Alivisatos, "Integration of colloidal nanocrystals into lithographically patterned devices," Nano Letters, vol. 4, no. 6, pp. 1093-1098, 2004.

[12] G. Mie, "Beitrage zur Optik truber Medien," Annals of Physics, vol. 25, pp. 377-445, 1908.

[13] S. Li, D. Wu, X. Xu, and R. Gu, "Theoretical and experimental studies on the adsorption behavior of thiophenol on gold nanoparticles," Journal of Raman Spectroscopy, vol. 38, no. 11, pp. 1436-1443, 2007.

[14] H. Xu, "Calculation of the near field of aggregates of arbitrary spheres," Journal of the Optical Society of America A, vol. 21, no. 5, pp. 804-809, 2004.

[15] H. X. Xu, "A new method by extending Mie theory to calculate local field in outside/inside of aggregates of arbitrary spheres," Physics Letters A, vol. 312, no. 5-6, pp. 411-419, 2003.

[16] L. Novotny and B. Hecht, Principles of Nano-Optics, Cambridge University Press, Cambridge, UK, 2006.

[17] J. Hu, B. Zhao, W. Xu, Y. Fan, B. Li, and Y. Ozaki, "Simple method for preparing controllably aggregated silver particle films used as surface-enhanced Raman scattering active substrates," Langmuir, vol. 18, no. 18, pp. 6839-6844, 2002.

[18] K. D. Alexander, M. J. Hampton, S. Zhang, A. Dhawan, H. $\mathrm{Xu}$, and R. Lopez, "A high-throughput method for controlled hot-spot fabrication in SERS-active gold nanoparticle dimer arrays," Journal of Raman Spectroscopy, vol. 40, no. 12, pp. 2171-2175, 2009.

[19] J. T. Krug, G. D. Wang, S. R. Emory, and S. Nie, "Efficient Raman enhancement and intermittent light emission observed in single gold nanocrystals," Journal of the American Chemical Society, vol. 121, no. 39, pp. 9208-9214, 1999.

[20] F. Huang and J. J. Baumberg, "Actively tuned plasmons on elastomerically driven Au nanoparticle dimers," Nano Letters, vol. 10, no. 5, pp. 1787-1792, 2010.

[21] P. Kambhampati, C. M. Child, M. C. Foster, and A. Campion, "On the chemical mechanism of surface enhanced Raman scattering: experiment and theory," The Journal of Chemical Physics, vol. 108, no. 12, pp. 5013-5027, 1998.
[22] S. Li, M. L. Pedano, S. H. Chang, C. A. Mirkin, and G. C. Schatz, "Gap structure effects on surface-enhanced Raman scattering intensities for gold gapped rods," Nano Letters, vol. 10, no. 5, pp. 1722-1727, 2010.

[23] W. Rechberger, A. Hohenau, A. Leitner, J. R. Krenn, B. Lamprecht, and F. R. Aussenegg, "Optical properties of two interacting gold nanoparticles," Optics Communications, vol. 220, no. 1-3, pp. 137-141, 2003.

[24] C. Tabor, R. Murali, M. Mahmoud, and M. A. El-Sayed, "On the use of plasmonic nanoparticle pairs as a plasmon ruler: the dependence of the near-field dipole plasmon coupling on nanoparticle size and shape," The Journal of Physical Chemistry A, vol. 113, no. 10, pp. 1946-1953, 2009.

[25] L. Gunnarsson, E. J. Bjerneld, H. Xu, S. Petronis, B. Kasemo, and M. Käll, "Interparticle coupling effects in nanofabricated substrates for surface-enhanced Raman scattering," Applied Physics Letters, vol. 78, no. 6, pp. 802-804, 2001.

[26] K. Bosnick, M. Maillard, L. Brus, and J. Jiang, "Single molecule Raman spectroscopy at the junctions of large ag nanocrystals," The Journal of Physical Chemistry B, vol. 107, no. 37, pp. 99649972, 2003.

[27] K. H. Su, Q. H. Wei, X. Zhang, J. J. Mock, D. R. Smith, and S. Schultz, "Interparticle coupling effects on plasmon resonances of nanogold particles," Nano Letters, vol. 3, no. 8, pp. 10871090, 2003.

[28] E. Prodan, C. Radloff, N. J. Halas, and P. Nordlander, "A hybridization model for the plasmon response of complex nanostructures," Science, vol. 302, no. 5644, pp. 419-422, 2003.

[29] P. Nordlander and E. Prodan, "Plasmon hybridization in nanoparticles near metallic surfaces," Nano Letters, vol. 4, no. 11, pp. 2209-2213, 2004. 

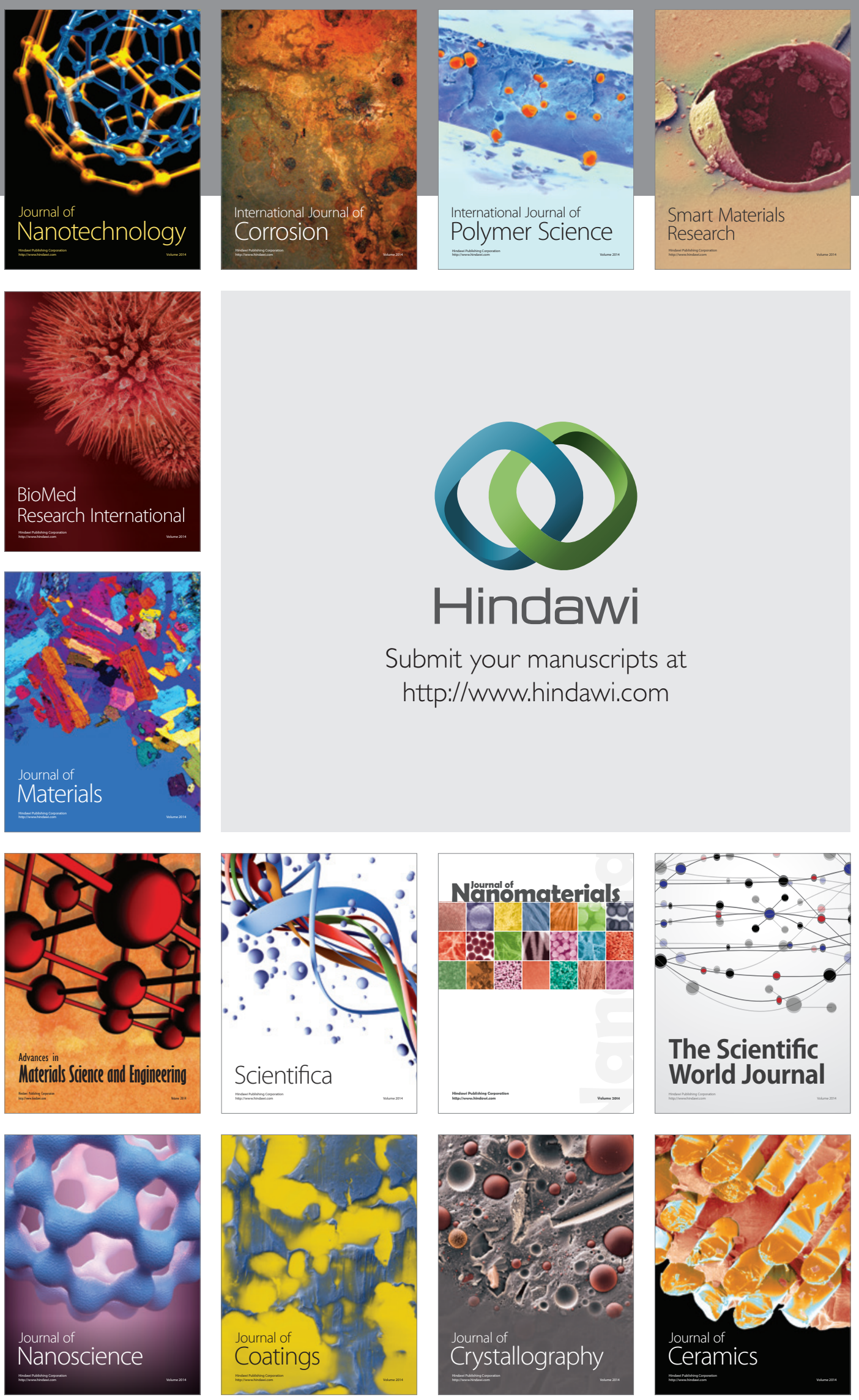

The Scientific World Journal

Submit your manuscripts at

http://www.hindawi.com

\section{World Journal}

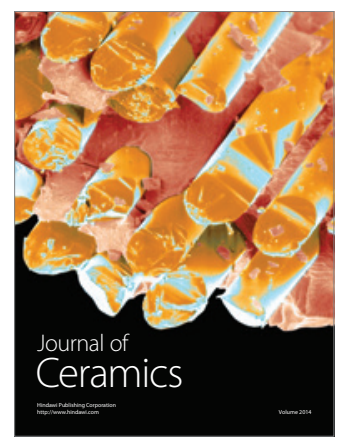

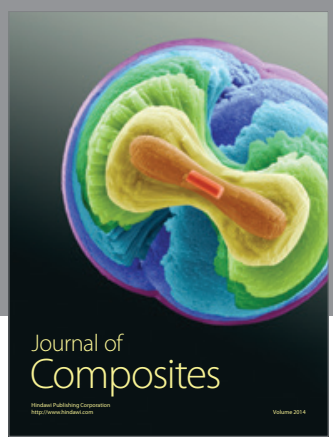
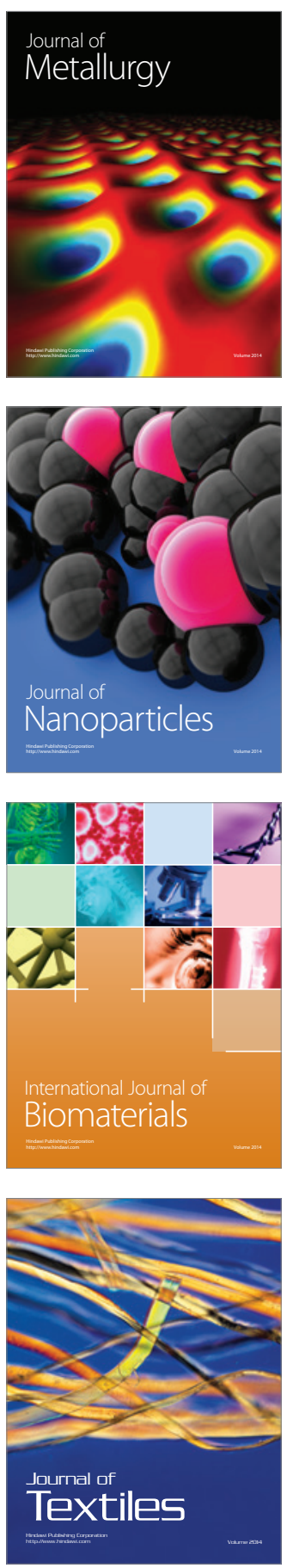